\title{
Accuracy Evaluation of Brain Tumor Detection using Entropy-based Image Thresholding
}

\author{
Amal Q. Alyahya \\ Middle East University/Faculty of Information Technology, Amman, Jordan \\ E-mail: amalalyahya@ meu.edu.jo \\ Ahmad A. Abu-Shareha \\ Middle East University/Faculty of Information Technology, Amman, Jordan \\ E-mail: aabushareha@meu.edu.jo
}

Received: 22 October 2017; Accepted: 08 January 2018; Published: 08 March 2018

\begin{abstract}
In this paper, the accuracy of the entropybased thresholding approaches in brain tumor detection framework is investigated. Entropies are information gain methods that have been used for image thresholding with various application and different image modalities. The accuracy of the existing entropies for image thresholding has been studied in general domain (e.g.: natural images) and were not compared thoroughly. Thus, a framework for brain tumor segmentation is proposed with the core process of the image thresholding, in order to evaluate the accuracy of the entropies. Five entropies, namely, Renyi, Maximum, Minimum, Tsallis and Kapur are evaluated. Moreover, the aggregation of entropies was implemented and evaluated. The results show that the maximum entropy is the best for brain tumor detection. Moreover, it was shown that aggregation of entropies output does not enhance the result, however, it works as automatic selection of the best result and produces the results with the highest accuracy.
\end{abstract}

Index Terms-Renyi Entropy, Tsallis Entropy, Maximum Entropy, Minimum Entropy, Image Segmentation, Thresholding, Brain Tumor Detection.

\section{INTRODUCTION}

Automatic tumor detection in an early stage is a critical task. Tumor is classified as a benign tumor, premalignant tumor and malignant. Benign tumor is the one that does not grow suddenly and has no effect on the other tissue, an example of this class of tumor is moles. Pre-malignant is the class that if it is not treated quickly, it becomes a malignant tumor. Malignant tumor grows rapidly and affects the neighboring tissue and, with time, it affects human life and leads to the death. Tumor detection is an important part of the treatment process. Thus, tumor detection techniques have concerned researchers in computer fields, especially, image processing [1].

One of the most important stages in tumor detection is image segmentation, in which tumor is being isolated from other healthy tissues. By isolating the tumor then determines its stage, the treatment becomes easier [2] Image thresholding is one of the techniques that are used for image segmentation [3]. Threshold techniques divide the image into two main regions, these are: Foreground and Background. The output of the thresholding process is a binary image with only two regions that formed by the highest possible contrast that could be found in the image [4]. This type of thresholding, which produce two regions, is called global thresholding. The other type of thresholding is called multi-thresholding, which is implemented by segmenting an image into multiple objects and background. Entropy is one of these approaches that aim at finding a threshold value that facilitates maximum information extraction from the image. Entropy has been emerged in Information Theory to extract the amount of information expressed by a piece of data [5].

Table 1. List of Entropies used in Image Thresholding

\begin{tabular}{|c|c|c|}
\hline Name & Aim & Usability \\
\hline Renyi & $\begin{array}{c}\text { Reduce error and } \\
\text { noise }\end{array}$ & $\begin{array}{c}\text { Works on the distribution of } \\
\text { gray level priorities that are } \\
\text { represented by the density scale. }\end{array}$ \\
\hline Maximum & $\begin{array}{c}\text { Reduce time and } \\
\text { increase equality }\end{array}$ & $\begin{array}{c}\text { Generate a strong correlation } \\
\text { between data partitions. }\end{array}$ \\
\hline Tsallis & Reduce time & $\begin{array}{c}\text { Determine the value of the gray- } \\
\text { level and mid-level gray in order } \\
\text { to choose the optimal data } \\
\text { distribution. }\end{array}$ \\
\hline Minimum & Noise resistant & $\begin{array}{c}\text { Increases the contrast at the } \\
\text { edges in the image. }\end{array}$ \\
\hline Kapur & $\begin{array}{c}\text { Reduce error and } \\
\text { noise }\end{array}$ & $\begin{array}{c}\text { Expresses quantifiable } \\
\text { information that gives the best } \\
\text { state of distribution. }\end{array}$ \\
\hline
\end{tabular}

Entropy is a Greece word, which means: if any system has many point of information's, the entropy finds equal distribution for this points that maximizes the information gain. Entropies not only used in computer sciences; it is used in many different fields, such as: physics, biology, astronomy, etc [6]. Entropy in image processing measures the amount of information that can be obtained from the image, either in its original form or after some processing. There are several ways to use entropy, as well as several 
equations to be used as the entropy basis [4]. The entropies that are used for thresholding, are many, each of them has a different aim, such as: reducing error, increase efficiency and remove noise. Various entropies are presented in the literature, the most commonly utilize entropies are listed in Table 1.

The accuracy of the existing entropies for image thresholding has been studied in general domain (e.g.: natural images) [7]. However, natural images are different from medical images by all means (e.g.: the contrast, colors, etc.). Moreover, medical images differ from each other by the means of organ and modality. Consequently, there is a need to evaluate the existing entropies for medical image segmentation [8].

The goal of this work is to evaluate the accuracy of the entropy-based thresholding approaches and their combination in brain tumor detection framework. This is implemented by: 1. developing a tumor detection framework that takes a brain image and produces a segmented image with a detected tumor if tumor is present, 2. using different entropy based thresholding in the developed tumor detection framework, 3. combining multiple thresholding approaches by applying logical operators (AND and OR) on the thresholding output and acquires an automatic selection of their outputs to get the best result and 4. evaluating the entropies results and their different combinations in the developed tumor detection framework. The rest of this paper is organized as follows: Section II discusses the related work in brain tumor detection and image processing using entropies. Section III presents the proposed framework for tumor detection and the utilized entropies. Section IV discusses the results. The conclusion is given in Section V.

\section{RELATED WORK}

Statistics say that the low survival rate of patients with a brain tumor is due to the lack of disease understanding. The most effective way for more success in dealing with the disease is the advances in medical image processing. However, brain images are complex and require careful processing stage in-order to reveal the underlying information. Accordingly, several approaches, techniques, and methods for brain tumor detection were proposed.

An approach for brain tumor detection using image thresholding has been proposed [9], which depends on a graph structure. After the threshold is applied, a graph structure of the brain regions in the image is generated. Based on the edge weights which reflect the region connectivity, the best option for tumor treatment is determined. These options are: surgery, radiation therapy and chemotherapy. The choice of therapy, for example depends on the size and type of tumor and its location, which all revealed in the constructed graph. Similarly, another approach for brain tumor extraction and segmentation from the MR images was proposed [10]. First, the image is enhanced. Then, k-means clustering is implemented over a group of different modality images that represent the same brain view.

For tumor identification, an approach that uses image segmentation was implemented [11]. The detected tumor is classified as benign tumor, pre-malignant tumor, and malignant tumor. This approach is claimed to help in the diagnose of a brain tumor at an early stage, which in turn prevent the disease to develop from benign into malignant. The framework is simple in the manner that depends on two stages, image segmentation, and region classification.

A fully an automatic tumor detection and quantifying framework using image thresholding was also proposed [12]. The framework consists of four main stages, these are: filtering, segmentation, tumor recognition and tumor analysis. The results show that the proposed framework has achieved a full result for the detection and analysis of tumor in MRI images, which is confirmed by a medical expert.

Several other approaches have proposed automatic detection of brain tumor based on using segmentation with other filtering processes [13-19], which are summarized in Table 2 .

There are many methods and techniques that are used the original entropies and enhanced entropies that are proposed in the literature for entropy-based image thresholding. The entropies, for image thresholding, are extended by modifying the underlying calculations, adding pre-processing or post-processing steps.

An image thresholding technique using Tsallis entropy was proposed [20]. The proposed approach extends the original entropy by proposing a two-dimensional histogram that captures the differences in neighborhood pixels. Image thresholding using Renyi entropy was proposed based on calculating the distribution of information between two regions. The final threshold value is the maximum value for the distribution of information components, which showed high efficiency and more accurate results [4].

Table 2. Summary of the Related Works of Brain Tumor Detection

\begin{tabular}{|c|c|c|}
\hline Ref. & Techniques & Results \\
\hline [9] & $\begin{array}{l}\text { Thresholding and Graph- } \\
\text { based Decision Making. }\end{array}$ & Effective tumor detection. \\
\hline [13] & $\begin{array}{l}\text { Used of priors and } \\
\text { logistical system. }\end{array}$ & Noise removal. \\
\hline [10] & $\begin{array}{l}\text { Principle filtering and K- } \\
\text { means clustering. }\end{array}$ & High detection rate. \\
\hline [14] & $\begin{array}{c}\text { K-means and } \\
\text { Morphology threshold }\end{array}$ & High quality segmentation. \\
\hline [15] & $\begin{array}{l}\text { Thresholding and noise } \\
\text { removal. }\end{array}$ & $\begin{array}{l}\text { MRI is better than CT for } \\
\text { tumor detection. }\end{array}$ \\
\hline$[12]$ & $\begin{array}{l}\text { Segmentation and region } \\
\text { analysis. }\end{array}$ & Effective tumor detection. \\
\hline [11] & $\begin{array}{l}\text { Threshold and watershed } \\
\text { segmentation }\end{array}$ & $\begin{array}{l}\text { Effective tumor detection for } \\
\text { the heterogeneous images. }\end{array}$ \\
\hline [16] & $\begin{array}{l}\text { Threshold and Canny } \\
\text { filter. }\end{array}$ & Effective tumor detection. \\
\hline [17] & Segmentation. & Efficient tumor detection. \\
\hline [18] & $\begin{array}{l}\text { Weighted aggregation } \\
\text { and classification. }\end{array}$ & Effective tumor detection. \\
\hline [19] & $\begin{array}{l}\text { Neighborhoods } \\
\text { relationships analysis. }\end{array}$ & $\begin{array}{l}\text { Remove noise and provide } \\
\text { primary estimates of the } \\
\text { tumor. }\end{array}$ \\
\hline
\end{tabular}

A new thresholding approach based on Tsallis entropy was proposed [23]. The proposed approach constructs a 
two-dimensional histogram by the gray value of all pixels compares with the average gray value of all pixels. Shannon entropies, which is identical to Renyi, was used to segment the image and highlight the edges [5].

A multi-level image thresholding based on Minimum entropy was also developed [21]. In-order to ease the process of calculating the distribution for all possible threshold values, the proposed approach uses an optimization technique. The experiments showed that using swarm optimization increases the efficiency of the minimum entropy. Another multi-level image thresholding based on Tsallis was also proposed [22]. Inorder to ease the process of calculating the distribution for all possible threshold values, the proposed approach uses an optimization approach. The experimental results showed that using Bee colony algorithm increases the efficiency of the Tsallis entropy.

Overall, different approaches were proposed for image processing based on using entropies for information extraction. The reviewed papers, above, shows that different entropies have shown to give different results in different domains and applications. Thus, there is no best entropy for all applications. The reviewed Literation is summarized in Table 3. In summary, brain tumor detection is implemented basically by segmenting the image into regions and recognize the tumor region, if present, in the image. One of the segmentation approaches is the thresholding, for image thresholding, different entropies were used. The entropies are either run directly on the image histogram or over features extracted from the image.

Table 3. Summary of the Related Works in Entropy-based Thresholding

\begin{tabular}{|c|c|l|c|c|c|c|c|c|}
\hline Ref. & Entropy & applications & $\begin{array}{c}\text { Feature- } \\
\text { based }\end{array}$ & Binarization & $\begin{array}{c}\text { Reduce } \\
\text { Error }\end{array}$ & $\begin{array}{c}\text { Enhance } \\
\text { Quality }\end{array}$ & $\begin{array}{c}\text { Reduce } \\
\text { Time }\end{array}$ & $\begin{array}{c}\text { Noise } \\
\text { Removal }\end{array}$ \\
\hline$[24]$ & Tsallis & Enhancement & & $\sqrt{ }$ & $\sqrt{ }$ & $\sqrt{ }$ & & \\
\hline$[20]$ & Tsallis & Segmentation & & $\sqrt{ }$ & & $\sqrt{ }$ & & \\
\hline$[25]$ & Maximum & Segmentation & & & $\sqrt{ }$ & $\sqrt{ }$ & $\sqrt{ }$ \\
\hline$[21]$ & Minimum & Segmentation & & & & $\sqrt{ }$ & $\sqrt{ }$ & \\
\hline$[4]$ & Renyi & Segmentation & $\sqrt{ }$ & $\sqrt{ }$ & & & & \\
\hline$[18]$ & Kapur & Segmentation & & $\sqrt{ }$ & & $\sqrt{ }$ & $\sqrt{ }$ & $\sqrt{ }$ \\
\hline$[23]$ & Tsallis & Segmentation & & $\sqrt{ }$ & & $\sqrt{ }$ & $\sqrt{ }$ & \\
\hline$[5]$ & Tsallis & Segmentation & & $\sqrt{ }$ & $\sqrt{ }$ & $\sqrt{ }$ & $\sqrt{ }$ & \\
\hline
\end{tabular}

\section{PROPOSED WORK}

A framework for brain tumor detection is built with a thresholding step, which is based on the entropy, is implemented as the main step in this framework. Besides thresholding, the proposed framework consists of several processing stages, as illustrated in Fig. 1.

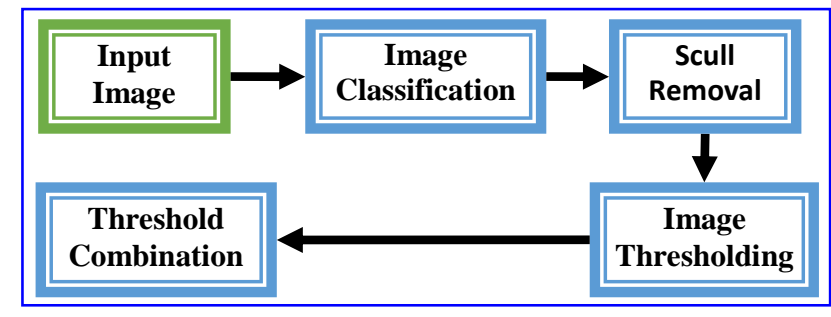

Fig.1. The Proposed Framework for Tumor Detection

The input to the framework is medical image, which should be for brain, MRI modality and gray-scale. Each pixel in gray-level images is represented by a single value of 0-255. The images are classified based on the presence and absence of tumor first then segmented for the purpose of tumor detection.

Image classification is implemented based on the images as a whole. Therefore, a set of training images with positive and negative labels (e.g.: presence or absence of tumor) where used with Support Vector Machine (SVM). The training and testing process for the classification task is implemented over the scaled image histogram, which are extracted prior to the classification process.

Scull removal, which is the process of excluding the outer structure of the brain, is implemented then on the images that are classified as positive with regards to the presence of the tumor. Scull removal helps in concentration in the interior region of the brain rather than worrying about the external unrelated parts. Technically, the skull is identified and removed from the complete circle with distinguishing color in the brain images. To get rid of this structure the white matter, gray and cerebrospinal fluid are isolated in the brain images using the level set method. An example of the input/output of the skull removal is given in Fig. 2.
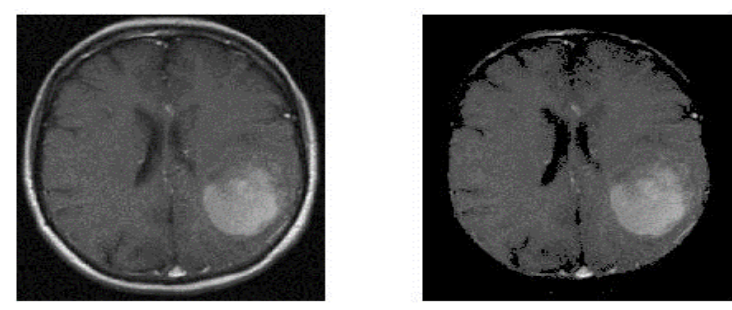

Fig.2. Scull Removal Example

The main component of the proposed work is the image thresholding. Thresholding takes as input the image of the brain and produces a threshold, or so called segmented image. Five types of entropies are used. The differences between the entropy were the calculations. Renyi entropy is based on mathematical equations, as given in (1), (2) and (3). 


$$
\begin{aligned}
& H 1(t)=\frac{1}{1-a} \ln \sum_{i=1}^{t} p_{i}^{a} \\
& H 2(t)=\frac{1}{1-a} \ln \sum_{j=t}^{n} p_{j}^{a} \\
& \text { T=MAX (H1 + H 2 ) }
\end{aligned}
$$

Tsallis entropy is based on mathematical equations, as given in (4), (5) and (6).

$$
\begin{gathered}
H 1_{n}^{x}(t)=\frac{1}{x-1} 1-\sum_{i=1}^{t} p_{i}^{x} \text { where } x \neq 0 \\
H 2_{n}^{x}(t)=1-\sum_{j=t}^{n} p_{i}^{x} \\
\mathrm{~T}=\operatorname{MAX}(\mathrm{H} 1+\mathrm{H} 2)
\end{gathered}
$$

Minimum entropy is seen as an extension of the maximum entropy, noted that in the absence of advanced sufficient information, both maximum and minimum produced preliminary equal information. Maximum entropy is calculated based on mathematical equations, as given in (7), (8) and (9).

$$
\begin{aligned}
& H 1(t)=-\sum_{i=1}^{t} P_{i} \log P_{i} \\
& H 1(t)=-\sum_{j=t}^{n} P_{j} \log P_{j} \\
& \text { T=MAX }(\mathrm{H} 1+\mathrm{H} 2)
\end{aligned}
$$

Kapur entropy is calculated based on mathematical equation, as given in (10), (11) and (12).

$$
\begin{aligned}
& H 1(t)=\frac{1-\left(\sum_{i=1}^{t} p_{i}^{1 / a}\right)^{a}}{1-a} \\
& H 1(t)=\frac{1-\left(\sum_{j=t}^{n} p_{j}^{1 / a}\right)^{a}}{1-a} \\
& \mathrm{~T}=\operatorname{MAX}(\mathrm{H} 1+\mathrm{H} 2)
\end{aligned}
$$

where, $\mathrm{H} 1$ and $\mathrm{H} 2$ are the generated parts using the threshold value, t. The parameter, a, is a small selected value in the range $(0-1), p_{i}$ and $p_{j}$ are the probability of data pieces in $\mathrm{H} 1$ and $\mathrm{H} 2$ regions, respectively.

The results of several thresholds, using different entropies, are combined. This work proposes a combination process based logical operators. Fig. 3, illustrates an example of such combination. The logic operators that are used, AND and OR, which are implemented as follows: The AND takes two inputs, which represents a corresponding pixel in the resulted segmented images from two entropies and produce one output.
In summary, brain tumor detection is implemented in the proposed work by, extracting features from the image, segmenting the image, using several thresholding and combined threshold process. The idea of entropy reflects the separation of objects from the background, which contributes significantly to the separation of tumor part from the rest of the brain discussed.

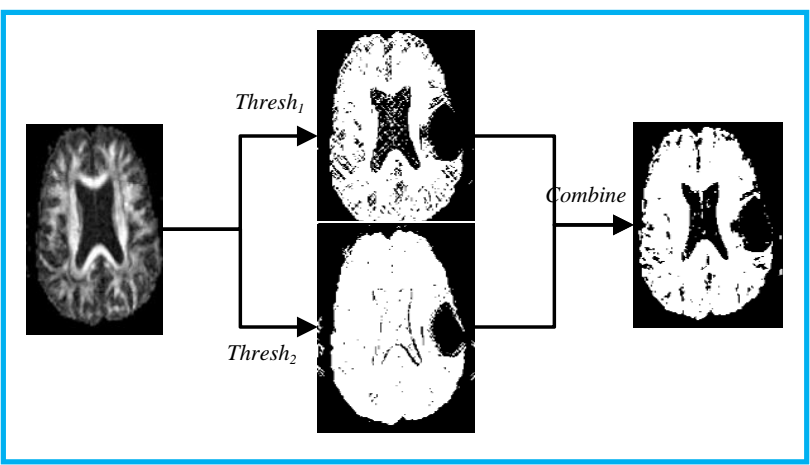

Fig.3. Threshold Combination

\section{RESULTS}

In order to experiment the proposed framework a set of brain images are collected. The dataset that is used are synthetic images mimics the natural brain images captured using the magnetic resonance images (MRI). Besides the images, the ground truth segmentation is provided for these images [9]. 300 images were used, 150 of these images are with tumor and 150 for healthy subjects. The resolutions of these images are 189x 188, the file size for each is in the range [17KB-30KB] and the type of images is PNG. The software used in the this work is MATLAB, a pilot program of mathematic programming and engineering calculations (Program version is R-2016). In this study, 5 entropy-based thresholding techniques have been applied to 150 different images with a brain tumor.

\section{A. Results of Individual Entropies}

The results of applying the proposed framework for tumor detection and identification on an input image is a segmented image with feasible and identified tumor region. The other tissues in the brain, the white matter and grey matter, are merged in the with the background. Using robust entropies, such as maximum entropy, the tumor is isolated from all other tissues. On the other hand, the results of applying a weak entropy on an image, such as minimum entropy, is a segmented image with unidentified tumor region, which is mixed with the other tissues, such as the white matters.

Fig. 4 illustrates the accuracy rate of the compared entropies using some of the images in the dataset. It can be clearly seen that maximum entropy gives the best results and minimum cross entropy has the lowest accuracy, as its best value the accuracy never reached $80 \%$, while all other methods have better accuracy rate. The average accuracy for these entropies is given in 
Table 4.

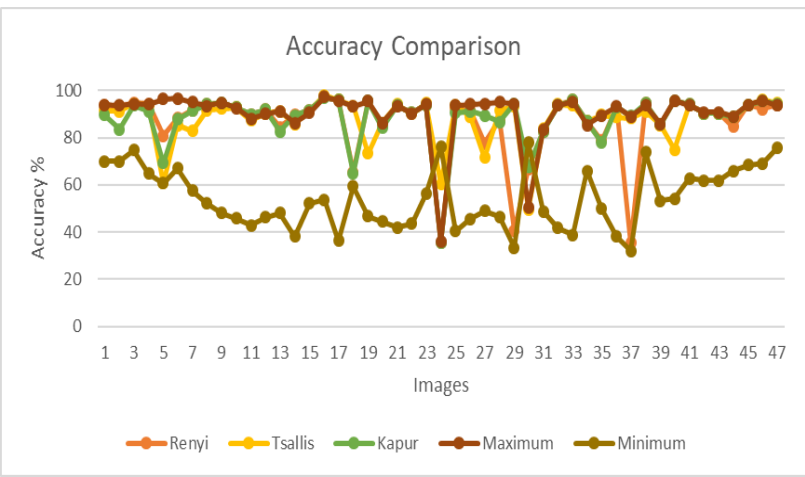

Fig.4. Entropies Comparison for Tumor Detection

Table 4. Average Accuracy of the Entropies

\begin{tabular}{|c|c|}
\hline Entropy & Accuracy \\
\hline Renyi & 86.35738753 \\
\hline Tsallis & 87.71731117 \\
\hline Kapur & 88.24548896 \\
\hline Maximum & $\mathbf{9 0 . 3 6 2 3 5 2 4 5}$ \\
\hline Minimum & 54.2981202 \\
\hline
\end{tabular}

\section{B. Results of Aggregated Entropies}

The result for applying AND and OR logical operators on the output images of two different entropies does not enhance the results. Nevertheless, using AND operator always produces an identical result with the entropy that has the best accuracy. On the other hand, OR operator always produces an identical result with the entropy that has the worst accuracy. Table 5 shows the cross matrix of the accuracy obtained by using AND with the underlying entropies, while Table 6 shows the results of using OR.

Table 5. Average Accuracy of the Entropies with AND

\begin{tabular}{|c|c|c|c|c|c|}
\hline AND & Renyi & Tsallis & Kapur & Max. & Min. \\
\hline Renyi & & 87.71 & 88.24 & $\mathbf{9 0 . 3 6}$ & 86.35 \\
\hline Tsallis & 87.71 & & 88.24 & $\mathbf{9 0 . 3 6}$ & 87.71 \\
\hline Kapur & 88.24 & 88.24 & & $\mathbf{9 0 . 3 6}$ & 88.24 \\
\hline Max. & $\mathbf{9 0 . 3 6}$ & $\mathbf{9 0 . 3 6}$ & $\mathbf{9 0 . 3 6}$ & & $\mathbf{9 0 . 3 6}$ \\
\hline Min. & 54.29 & 87.71 & 88.24 & $\mathbf{9 0 . 3 6}$ & \\
\hline
\end{tabular}

Table 6. Average Accuracy of the Entropies with OR

\begin{tabular}{|c|c|c|c|c|c|}
\hline OR & Renyi & Tsallis & Kapur & Max. & Min. \\
\hline Renyi & & 86.35 & 86.35 & 86.35 & 54.29 \\
\hline Tsallis & 86.35 & & 87.71 & 87.71 & 54.29 \\
\hline Kapur & 86.35 & 87.71 & & 88.24 & 54.29 \\
\hline Max. & 86.35 & 87.71 & 88.24 & & 54.29 \\
\hline Min. & 54.29 & 54.29 & 54.29 & 54.29 & \\
\hline
\end{tabular}

An example of the output results is given in Figure 5. Fig. 5(A) illustrates a brain with the tumor as captured by Magnetic Resonance. Fig. 5(B) is the image after applying minimum cross entropy thresholding. Fig. 5(C) gives the result of applying maximum entropy

thresholding and Fig. 5(D) is the result of merge the output of two entropies. Figure 6 illustrates an example using the OR operator. The results of AND operator, as illustrated in Fig. 5 and Figure 7, is a selection of the darkest pixel, region, segment among the underlying input images. Subsequently, AND operator, when applied with one or more good input will produce good output, because it identifies the tumor and wrap the rest with the background. While, the results of OR operator as illustrated in Fig. 6 and Fig. 8, is a selection of the brighter pixel, region, segment among the underlying input images. Subsequently, the results wrap the tumor with the white matter, which results in bad output.

Ddetailed comparison between the involved entropies and their aggregation, for the first 47 images in the utilized dataset, is given in Appendix A.

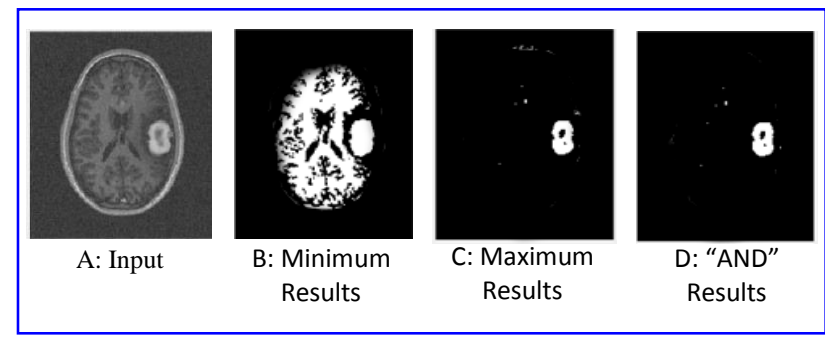

Fig.5. Minimum and Maximum Aggregation using “AND” Operator

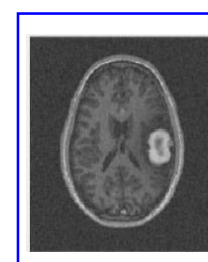

A: Input

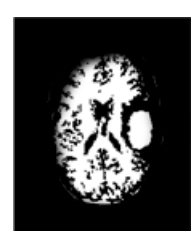

B: Minimum Results

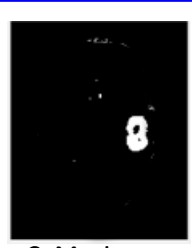

C: Maximum Results

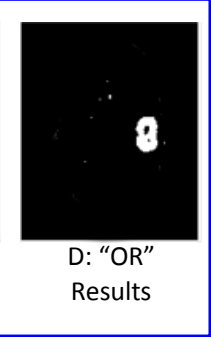

Fig.6. Minimum and Maximum Aggregation using "OR” Operator

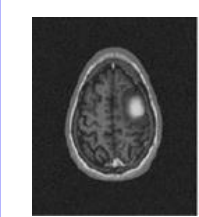

A: Input

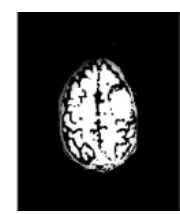

B: Minimum Results

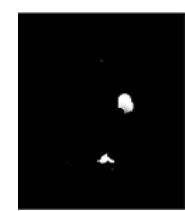

C: Renyi

Results

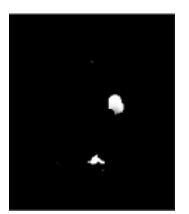

D: “AND" Results

Fig.7. Minimum and Renyi Aggregation using "AND” Operator

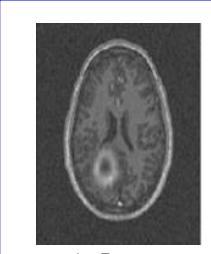

A: Input

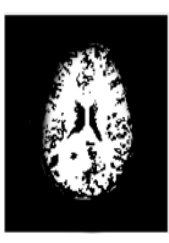

B: Minimum Results
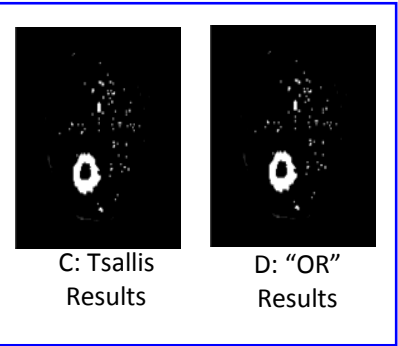

Fig.8. Minimum and Renyi Aggregation using "OR" Operator 


\section{CONCLUSION}

In this paper, an evaluation of the entropies accuracy and their combination in brain tumor detection has been implemented. A framework is proposed for tumor detection that takes as input brain image and produces a segmented image with a detected tumor if tumor is presented. The brain tumor detection is implemented in the proposed work by, remove the scull from the image, segmenting the image, using several thresholds and combined threshold process. It was proven that entropy separate objects from the background, which contributes significantly to the separation of tumor from the rest of the brain. The developed framework uses different entropy based thresholding and compared between different entropies based thresholding. The results showed that maximum entropy achieved the best result in the tumor detection framework. The aggregation of the entropies using AND operations has played the roles of automatic selection of the best threshold.

\section{APPENDIX A}

More results are illustrated in Fig. A.1, Fig. A.2, Fig. A.3, Fig. A.4, Fig. A.5, Fig. A.6, Fig. A.7, Fig. A.8, Fig. A.9 and Fig. A.10.

\section{Min and Max}

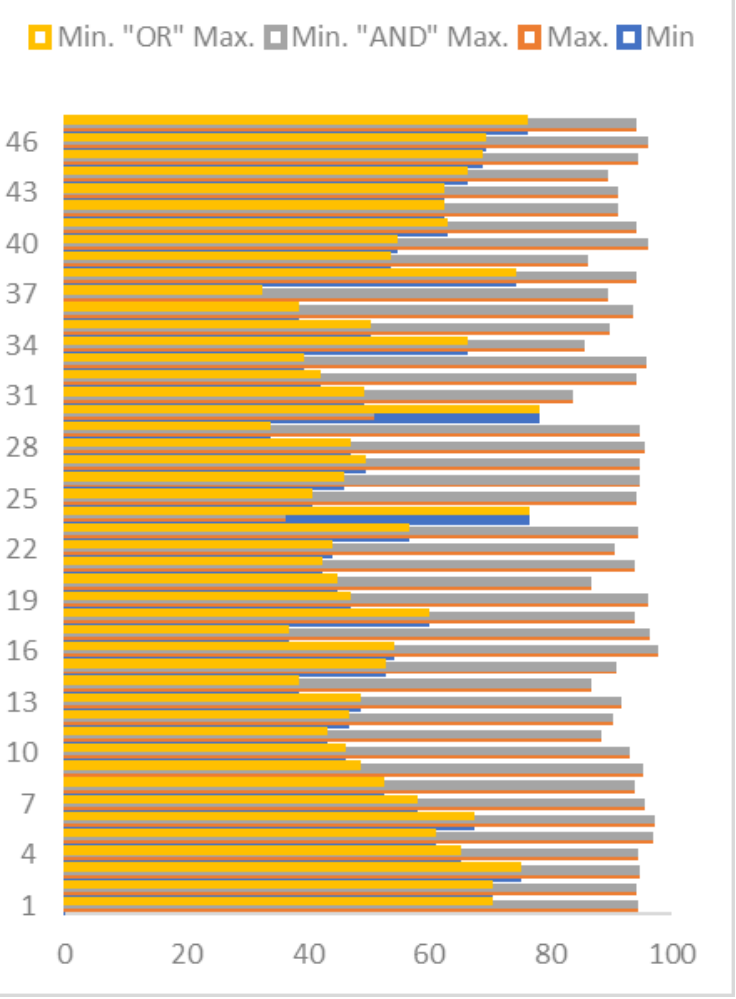

Fig.A.1. Min and Max Results

\section{Min and Kapur}

ㅁMin. "OR" Kapur ㅁMin. "AND" Kapur Kapur םMin

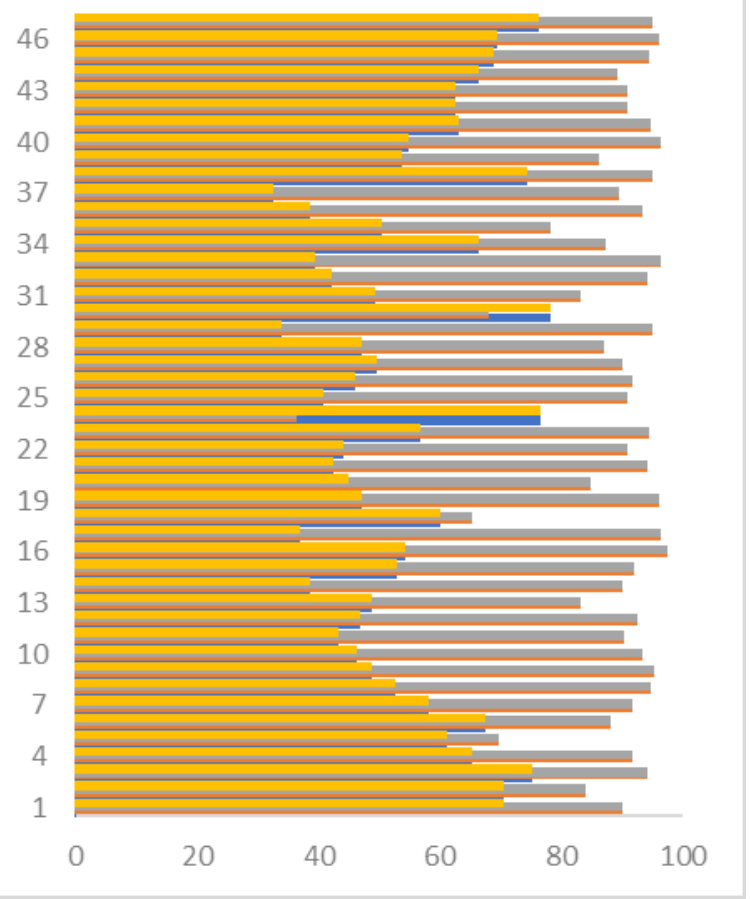

Fig.A.2. Min and Kapur Results

\section{Min and Tsallis}

口Min. "OR" Tsallis 口Min. "AND" Tsallis םTsallis $\mathbf{\square}$ Min

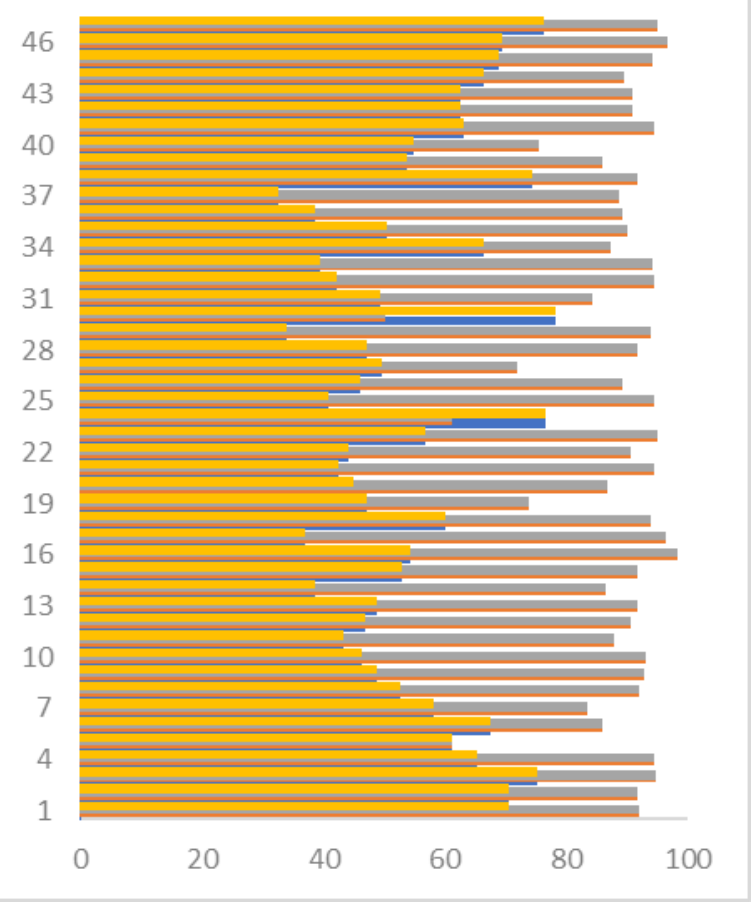

Fig.A.3. Min and Tsallis Results 


\section{Min and Renyi}

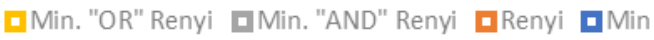

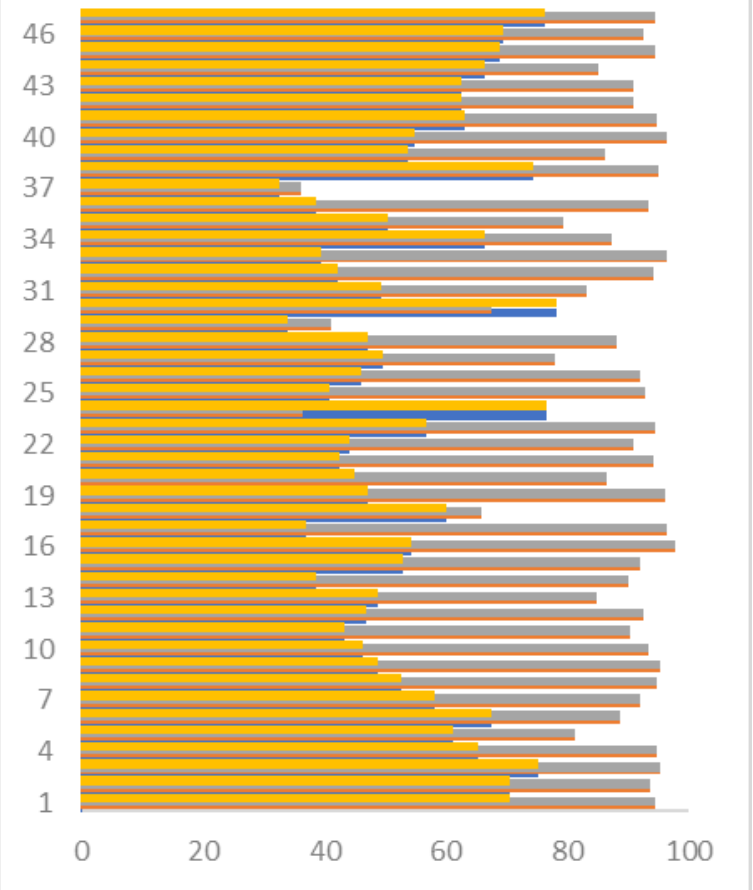

Fig.A.4. Min and Renyi Results

\section{Max and Kapur}

口Max. "OR" Kapur 口Max. "AND" Kapur םKapur םMax.

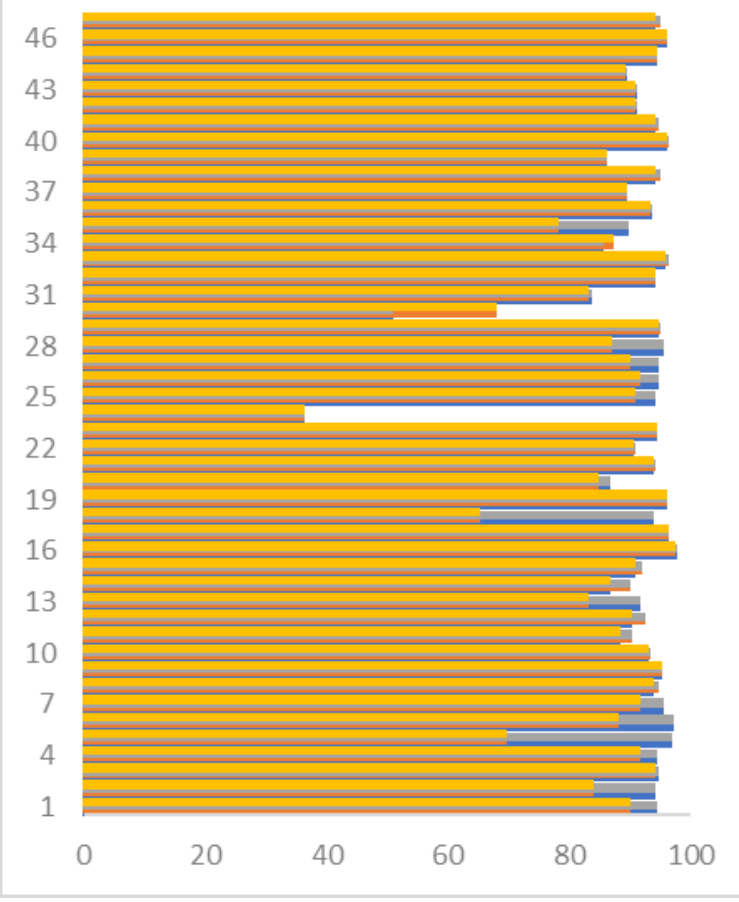

Fig.A.5. Max and Kapur Results

\section{Max and Tsallis}

口Max. "OR" Tsallis पMax. "AND" Tsallis 口Tsallis $\mathbf{\square}$ Max.

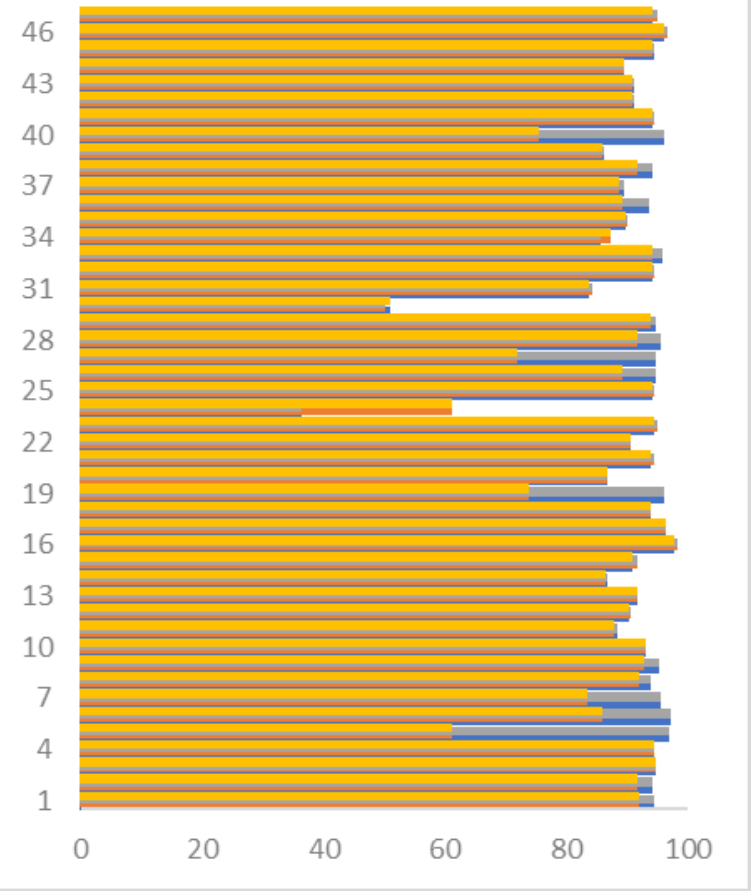

Fig.A.6. Max and Tsallis Results

\section{Max and Renyi}

口Max. "OR" Renyi 口Max. "AND" Renyi םRenyi םMax.

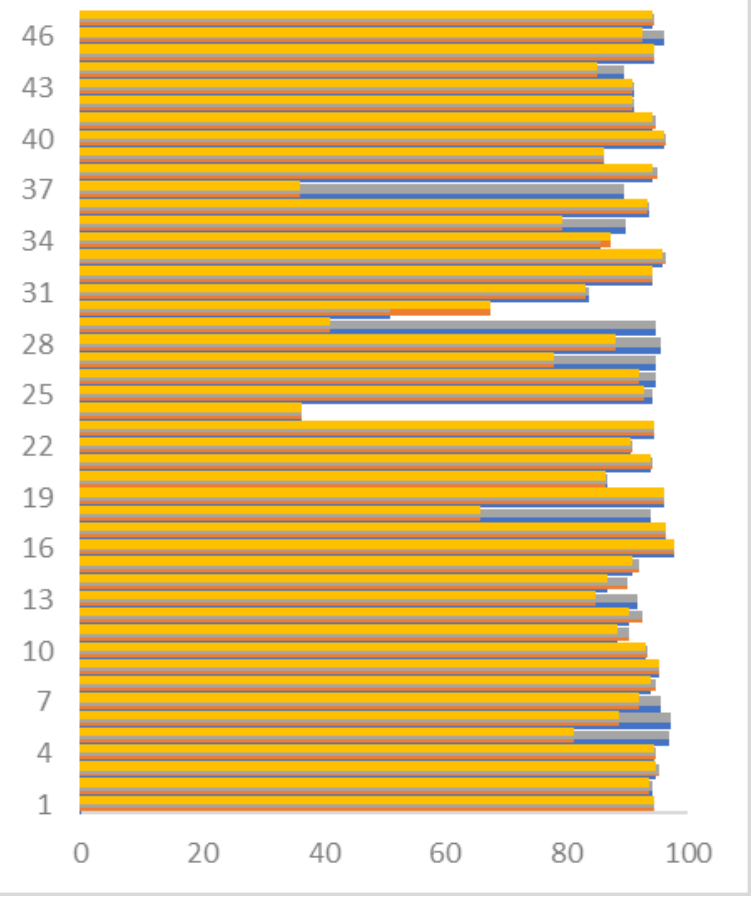

Fig.A.7. Max and Renyi Results 


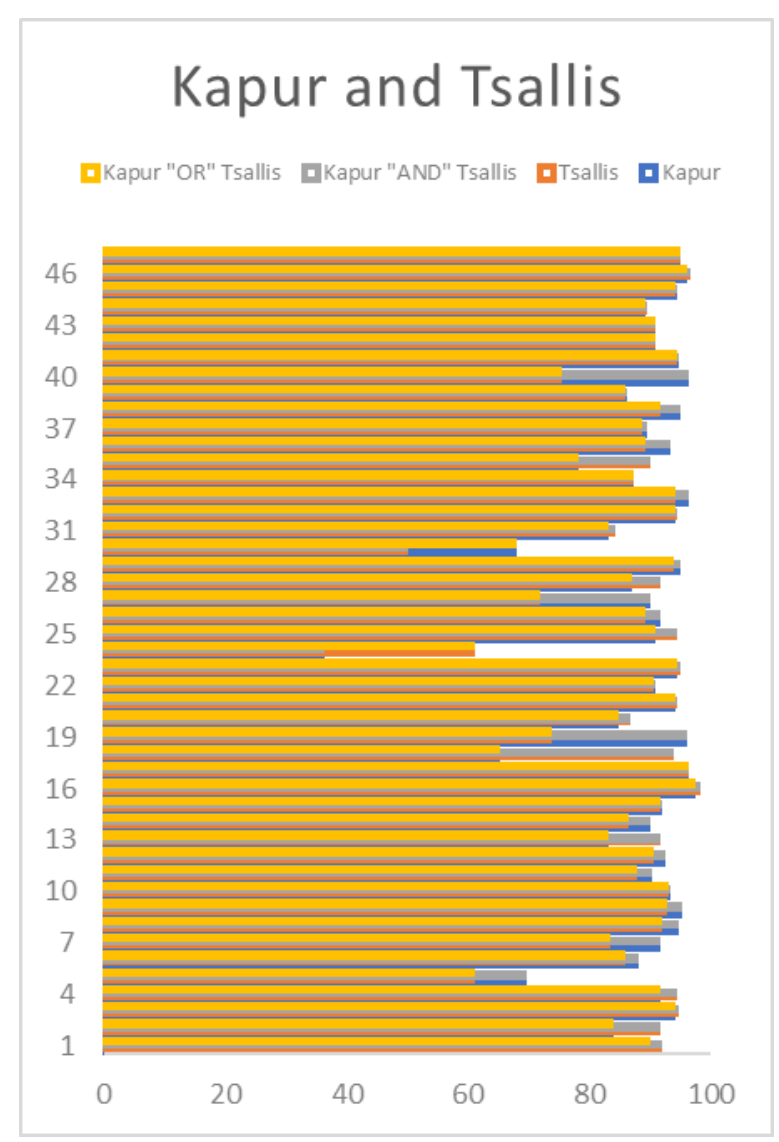

Fig.A.8. Kapur and Tsallis Results

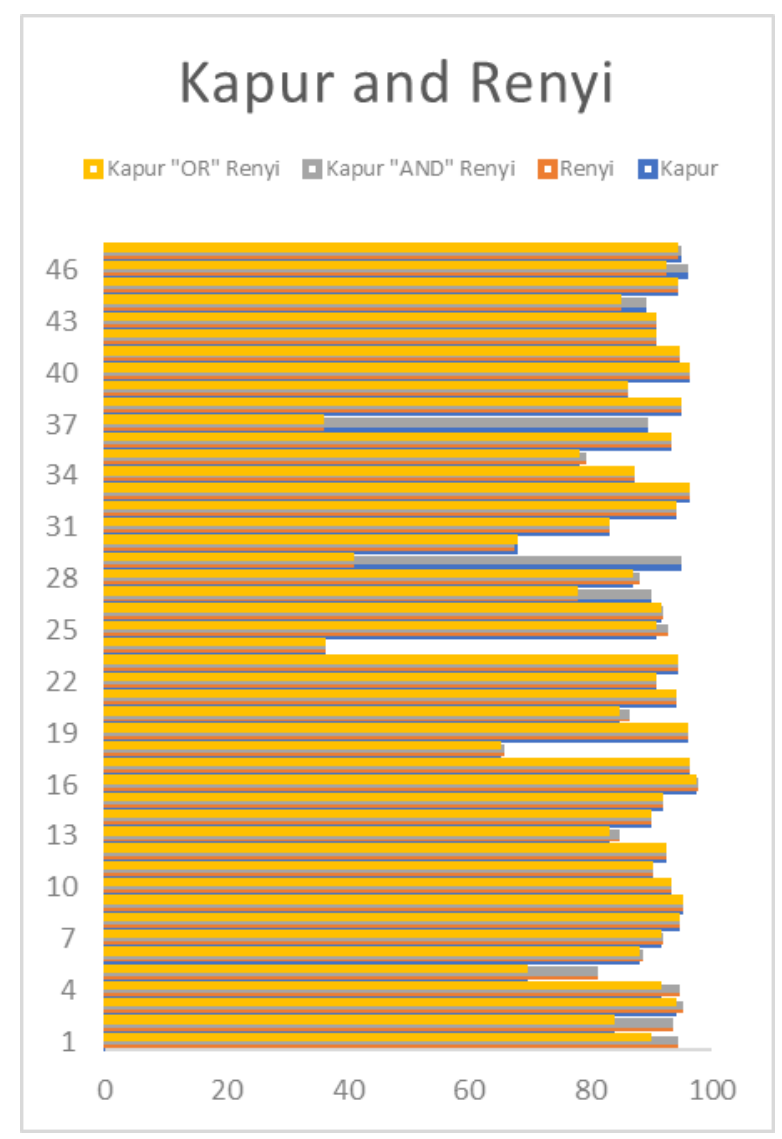

Fig.A.9. Kapur and Renyi Results

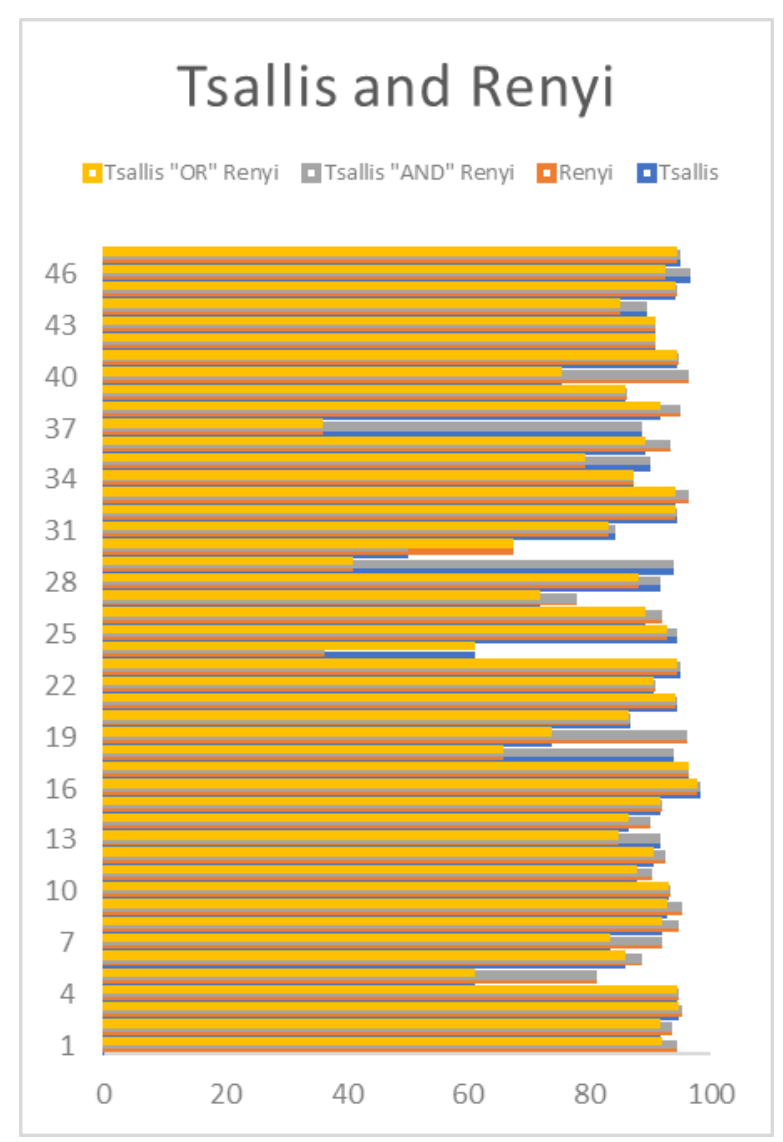

Fig.A.10. Tsallis and Renyi Results

\section{REFERENCES}

[1] M.-N. Wu, C.-C. Lin, and C.-C. Chang, "Brain tumor detection using color-based k-means clustering segmentation," in Intelligent Information Hiding and Multimedia Signal Processing, 2007. IIHMSP 2007. Third International Conference on, 2007, vol. 2, pp. 245-250: IEEE.

[2] S. J. Sangwine and R. E. Horne, The colour image processing handbook. Springer Science \& Business Media, 2012.

[3] Z. Ye, M. Wang, H. Jin, W. Liu, and X. Lai, "An Image Thresholding Approach Based on Ant Colony Optimization Algorithm Combined with Genetic Algorithm," International Journal of Intelligent Systems and Applications, vol. 7, no. 5, p. 8, 2015.

[4] A. A. A. Shareha, M. Rajeswari, and D. Ramachandram, "Textured Renyi Entropy for Image Thresholding," in Computer Graphics, Imaging and Visualisation, 2008. CGIV'08. Fifth International Conference on, 2008, pp. 185-192: IEEE.

[5] M. A. El-Sayed, "A new algorithm based entropic threshold for edge detection in images," arXiv preprint arXiv:1211.2500, 2012.

[6] L. Wang and Y. Chen, "Diversity based on entropy: A novel evaluation criterion in multi-objective optimization algorithm," International Journal of Intelligent Systems and Applications, vol. 4, no. 10, p. 113, 2012.

[7] D. Zhao, Y. Chan, and W. Gao, "Low-complexity and low-memory entropy coder for image compression," IEEE Transactions on Circuits and Systems for Video Technology, vol. 11, no. 10, pp. 1140-1145, 2001.

[8] M. Khalil, "Medical Image Steganography: Study of 
Medical Image Quality Degradation when Embedding Data in the Frequency Domain," International Journal of Computer Network and Information Security, vol. 9, no. 2, p. 22, 2017.

[9] M. Prastawa, E. Bullitt, and G. Gerig, "Simulation of brain tumors in MR images for evaluation of segmentation efficacy," Medical image analysis, vol. 13, no. 2, pp. 297-311, 2009.

[10] M. M. Ahmed and D. B. Mohamad, "Segmentation of brain MR images for tumor extraction by combining kmeans clustering and perona-malik anisotropic diffusion model," International Journal of Image Processing, vol. 2, no. 1, pp. 27-34, 2008.

[11] A. Mustaqeem, A. Javed, and T. Fatima, "An efficient brain tumor detection algorithm using watershed \& thresholding based segmentation," International Journal of Image, Graphics and Signal Processing, vol. 4, no. 10, p. 34, 2012.

[12] S. Roy and S. K. Bandyopadhyay, "Detection and Quantification of Brain Tumor from MRI of Brain and it's Symmetric Analysis," International Journal of Information and Communication Technology Research, vol. 2, no. 6, 2012.

[13] D. Cobzas, N. Birkbeck, M. Schmidt, M. Jagersand, and A. Murtha, "3D variational brain tumor segmentation using a high dimensional feature set," in Computer Vision, 2007. ICCV 2007. IEEE 11th International Conference on, 2007, pp. 1-8: IEEE.

[14] A. Kharrat, N. Benamrane, M. B. Messaoud, and M. Abid, "Detection of brain tumor in medical images," in Signals, Circuits and Systems (SCS), 2009 3rd International Conference on, 2009, pp. 1-6: IEEE.

[15] M. U. Akram and A. Usman, "Computer aided system for brain tumor detection and segmentation," in Computer Networks and Information Technology (ICCNIT), 2011 International Conference on, 2011, pp. 299-302: IEEE.

[16] S. Xavierarockiaraj, K. Nithya, and R. M. Devi, "Brain tumor detection using modified histogram thresholdingquadrant approach," Journal of Computer Applications (JCA), vol. 5, no. 1, pp. 21-25, 2012.

[17] S. Bauer, R. Wiest, L.-P. Nolte, and M. Reyes, "A survey of MRI-based medical image analysis for brain tumor studies," Physics in medicine and biology, vol. 58, no. 13, p. R97, 2013.

[18] A. K. Bhandari, V. K. Singh, A. Kumar, and G. K. Singh, "Cuckoo search algorithm and wind driven optimization based study of satellite image segmentation for multilevel thresholding using Kapur's entropy," Expert Systems with Applications, vol. 41, no. 7, pp. 3538-3560, 2014.

[19] B. H. Menze et al., "The multimodal brain tumor image segmentation benchmark (BRATS)," IEEE transactions on medical imaging, vol. 34, no. 10, pp. 1993-2024, 2015.

[20] P. K. Sahoo and G. Arora, "Image thresholding using twodimensional Tsallis-Havrda-Charvát entropy," Pattern recognition letters, vol. 27, no. 6, pp. 520-528, 2006.

[21] P.-Y. Yin, "Multilevel minimum cross entropy threshold selection based on particle swarm optimization," Applied mathematics and computation, vol. 184, no. 2, pp. 503513, 2007.

[22] Y. Zhang and L. Wu, "Optimal multi-level thresholding based on maximum Tsallis entropy via an artificial bee colony approach," Entropy, vol. 13, no. 4, pp. 841-859, 2011.

[23] M. A. El-Sayed, S. Abdel-Khalek, and E. Abdel-Aziz, "Study of efficient technique based on 2D tsallis entropy for image thresholding," arXiv preprint arXiv:1401.5098, 2014.
[24] C.-I. Chang, K. Chen, J. Wang, and M. L. Althouse, "A relative entropy-based approach to image thresholding," Pattern recognition, vol. 27, no. 9, pp. 1275-1289, 1994.

[25] S. J. Phillips, R. P. Anderson, and R. E. Schapire, "Maximum entropy modeling of species geographic distributions," Ecological modelling, vol. 190, no. 3, pp. 231-259, 2006.

\section{Authors' Profiles}

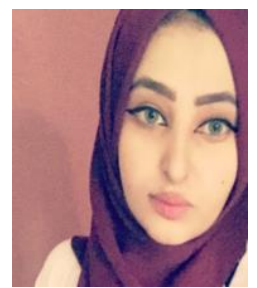

Amal Q. Alyahya is currently a $\mathrm{PhD}$ student at The University of Jordan. She received her first degree in computer engineer from Fahad Bin Sultan University, Saudi Arabia in 2014 and her master degree in Computer Science from Middle East University, Jordan.

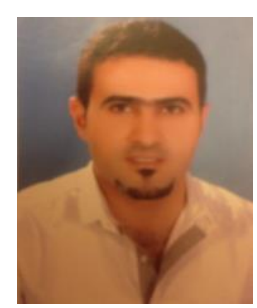

Ahmad A. Abu-Shareha is an assistant professor and the head of departments of Computer Information Systems (CIS) in Middle East University (MEU). He received his first degree in Computer Science from Al Al-Bayt University (AABU), Jordan, 2004, his Master degree from Universiti Sains Malaysia (USM) Malaysia, 2006 and his Ph.D degree from Universiti Sains Malaysia (USM) - Malaysia, 2012. His research focuses on Data mining, artificial intelligent and Multimedia Security.

How to cite this paper: Amal Q. Alyahya, Ahmad A. AbuShareha, "Accuracy Evaluation of Brain Tumor Detection using Entropy-based Image Thresholding", International Journal of Information Technology and Computer Science(IJITCS), Vol.10, No.3, pp.9-17, 2018. DOI: 10.5815/ijitcs.2018.03.02 